\title{
Expression and Significance of MicroRNA155 in Serum of Patients with Cerebral Small Vessel Disease
}

\author{
Ying Guo, Dongxue Li, Jiapei Li, ${ }^{2}$ Nan Yang, ${ }^{1}$ Deyun Wang ${ }^{1}$ \\ Department of Neurology, ${ }^{\dagger}$ Pu'er People's Hospital, Pu'er, China \\ Department of Internal Medicine, ${ }^{2}$ Pu'er City Prison Hospital, Pu'er, China
}

Objective : This study aimed to investigate the changes and significance of microRNA155 levels in serum of patients with cerebral small vessel disease (CSVD).

Methods : Thirty patients with CSVD who met the inclusion criteria were selected and divided into eight patients with lacunar infarction (LI) group and 22 patients with multiple lacunar infarction (MLI) combined with white matter lesions (WML) group according to the results of head magnetic resonance imaging (MRI). Thirty samples from healthy volunteers without abnormalities after head MRI examination were selected as the control group. The levels of serum microRNA155 in each group were determined by real-time polymerase chain reaction, and the correlation between microRNA155 in the serum of patients with CSVD and the increase of imaging lesions was analyzed by Spearman correlation analysis.

Results : Compared with the control group, the serum microRNA155 level in the LI group, MLI combined with WML group increased, the difference was statistically significant $(p<0.05)$; serum microRNA155 level was positively correlated with the increase of imaging lesions $(p<0.05)$.

Conclusion : The change of serum microRNA155 level in patients with CSVD may be one of its self-protection mechanisms, and the intensity of this self-protection mechanism is positively correlated with the number of CSVD lesions.

Key Words : Cerebral small vessel disease $\cdot$ Serum $\cdot$ microRNA155 level $\cdot$ Real-time polymerase chain reaction $\cdot$ Self protection mechanism.

\section{INTRODUCTION}

Cerebral small vessel disease (CSVD) is a common cerebrovascular disease ${ }^{17)}$. With the continuous development of imaging, the detection rate of CSVD has also been increasing ${ }^{31)}$. Some studies have found that CSVD is closely related to Alzheimer disease (AD) and Parkinson's disease. There is a pathophysiological synergistic effect between AD and CSVD. And there is also a relationship between CSVD and mild Parkinson's sign, the latter caused by the former with interfering the basal ganglia-thalamic cortical circuit involving the frontal and parietal lobes ${ }^{5}$.

Hypertension or diabetes is important in the development of CSVD, but the exact pathogenesis of CSVD is still un-

- Received : August 16, 2019 •Revised : November 8, 2019 •Accepted : November 30, 2019

- Address for reprints : Ying Guo

Department of Neurology, Pu'er People's Hospital, No. 44, Zhenxing Avenue, Simao District, Puer City, Yunnan Province 665000, China Tel : +86-18087991480, Fax : +86-0879-2131802, E-mail : guoyings1225@163.com, ORCID : https://orcid.org/0000-0001-7448-4552 
clear $^{26)}$. It has been found that increased blood-brain barrier (BBB) permeability and endothelial dysfunction are associated with CSVD, and the destruction of BBB is an important pathological feature of CSVD. Therefore, circulating biomarkers of vascular endothelial dysfunction may play an important role in CSVD recognition ${ }^{18,25)}$. The high expression of endothelial markers, such as intracellular adhesion molecule-1, has been proved to be related to WMH progression and and supports the role of endothelial dysfunction in $\mathrm{CSVD}^{13)}$. Associations between inflammatory markers and CSVD have also been reported in many population-based cohort studies.

MicroRNA-155 (miR-155) plays an important role in a variety of cellular functions, including hematocyte differentiation, immunity, inflammation and cardiovascular disease. MicroRNAs (miRs) is a typical post-transcriptional gene expression regulator, which has significant stability in serum ${ }^{21)}$. MiR155 is an important member in miRs, which is vital to body function, involving hematopoietic cells, immunity, inflammation and cardiovascular disease $\mathrm{7}^{7,11,22,24)}$. In addition, miR155 , as an oncogene, has over expression in a variety of malignant tumors, including nasopharyngeal carcinoma, breast cancer, hepatocellular carcinoma and gastric cancer. According to reports, hippocampal dysfunction is associated with depression $^{23,28,33,35,37)}$. In recent years, the role and mechanism of miR-155 in CSVD is still unclear. And the research on CSVD is mostly risk factor analysis and imaging changes, but there are few reports on whether there are self-protection mechanisms and self-protection mechanisms research.

\section{MATERIALS AND METHODS}

\section{Materials}

The study was approved by the Ethics Committee of Pu'er People's Hospital, and all subjects signed an informed consent form. Thirty patients with CSVD who met the inclusion criteria in the Department of Neurology, Pu'er People's Hospital from October 2018 to November 2018 were selected, all of which have had the complete magnetic resonance imaging (MRI) and susceptibility weighted imaging (SWI) of the crania. According to the result of cranial MRI examination, they were divided into lacunar infarction (LI) group and multiple lacunar infarction (MLI) combined with white matter lesions (WML) group (Fig. 1). Thirty samples from healthy volunteers without abnormalities after head MRI examination of Pu'er People's Hospital were selected as the control group. There were eight patients with LI, including six males and two females, aged 49-68 years old (58.72 \pm 7.34$) ; 22$ patients with MLI combined with WML, 15 males and seven females, aged $48-71$ years old (60.04 \pm 7.08$)$; healthy volunteers in the control group of 20 males and 10 females, aged 48-69 years old (59 \pm 6.71$)$. There were no significant differences between the three groups in gender and age $(p<0.05)$, which were comparable.

\section{Diagnostic criteria}

At present, there is no unified conclusion on the diagnostic criteria for CSVD. This study refers to the "Chinese CSVD di-

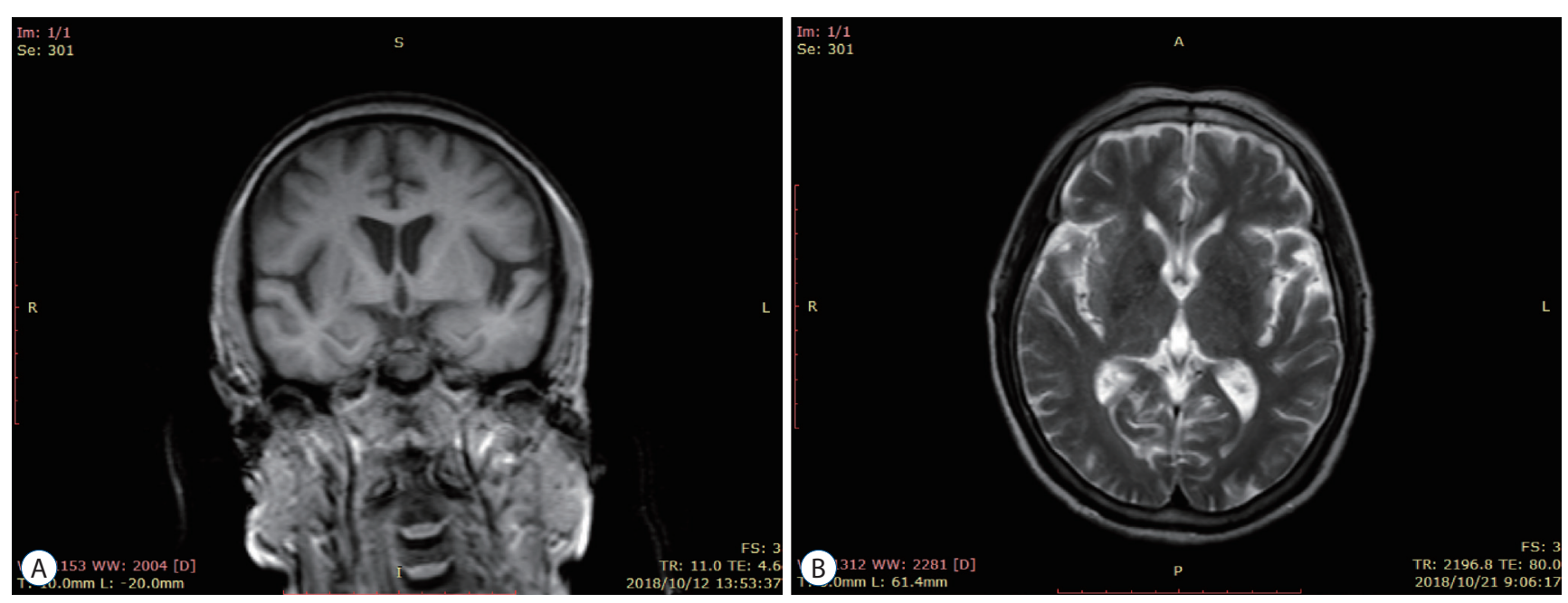

Fig. 1. Magnetic resonance imaging manifestations of patients. A : Lacunar infarction group. B : Multiple lacunar infarction combined with white matter lesions group. 
agnosis and treatment consensus ${ }^{\text {"3) }}$ and combined with the common performance of MRI to establish the following criteria : 1) LI : MRI showed a circular, elliptical or fissure-like cavity with a clear boundary, 3-15 $\mathrm{mm}$ in diameter, a significantly low signal on T1WI and a high signal on T2WI (Fig. 1A); 2) WML : manifested as punctate or flaky abnormal signals in the basal ganglia, semi-oval center, and radiation crown, T1WI showed low signal, T2WI and MRI liquid attenuation inversion recovery sequence was high signal, lesion boundary was blurred and the diameter was more than $5 \mathrm{~mm}$ (Fig. 2A); 3) cerebral microbleeds : MRI T2WI gradient echo or SWI showed uniform circular low-signal lesion with a diameter of 2-5 mm, surrounded by no edema (Fig. 2B); and 4) expansion of the perivascular space : T2WI had a high signal, diameter $<3 \mathrm{~mm}$, circular or linear, located in white matter and deep gray matter, and a low signal was visible on T1 (Fig. 2C).

\section{Inclusion criteria and exclusion criteria}

Inclusion criteria : 1) those with complete MRI and SWI data; 2) those who meet the above criteria for diagnosis of CSVD (diagnostic criteria 1 and/or diagnostic criteria 2); 3) MRI indicates that the infarct is LI or MLI combined with WML; and 4) those who have no communication problems and agree to participate in the study.

Exclusion criteria : 1) MRI showed that the diameter of the infarct was $>20 \mathrm{~mm}$; 2) computed tomography angiography of the head and neck showed stenosis of the intracranial and extracranial aorta, intracranial hemorrhagic disease or previous cerebral hemorrhage, cardiogenic cerebral embolism; 3) those with tumors, severe heart, lung, liver and kidney diseases and blood diseases; 4) those with genetic, metabolic, poisoning and rheumatic immune system diseases; and 5) those with central nervous system infections, multiple sclerosis and other central systems inflammatory demyelinating diseases, brain trauma, brain tumors, etc.

\section{Sample collection and detection}

In the early morning, the peripheral arterial blood of the elbow artery was taken on fasting, and the blood sample was stored in a centrifuge tube containing heparin. After the sample was collected for 30 minutes, it was centrifuged at 2000 $\mathrm{r} / \mathrm{min}$ at $4^{\circ} \mathrm{C}$ for 15 minutes. The serum was separated and stored in a refrigerator at $-80^{\circ} \mathrm{C}$ for reserve. Real-time quantitative polymerase chain reaction was used to detect the expression level of miR-155 in serum. U6 RNA primers (internal reference primers) and miR-155 stem-loop primers were purchased from Guangzhou RiboBio Co., Ltd (Guangzhou, China). The relative quantity of gene expression was expressed by $2^{-\Delta \Delta C t}$.

\section{Statistical analysis}

SPSS ver. 20.0 statistical software (IBM, Guangzhou, Guangdong, China) was used for data analysis. The normal distribution of measurement data was expressed by mean \pm standard deviation. Analysis of variance was used. Two independent samples $t$ test were used for comparison between the two groups, and Spearman correlation analysis was adopted. $p<0.05$ was considered statistically significant.

\section{RESULTS}

\section{MiR-155 levels in the three groups of serum}

Compared with the control group, the levels of miR-155 in
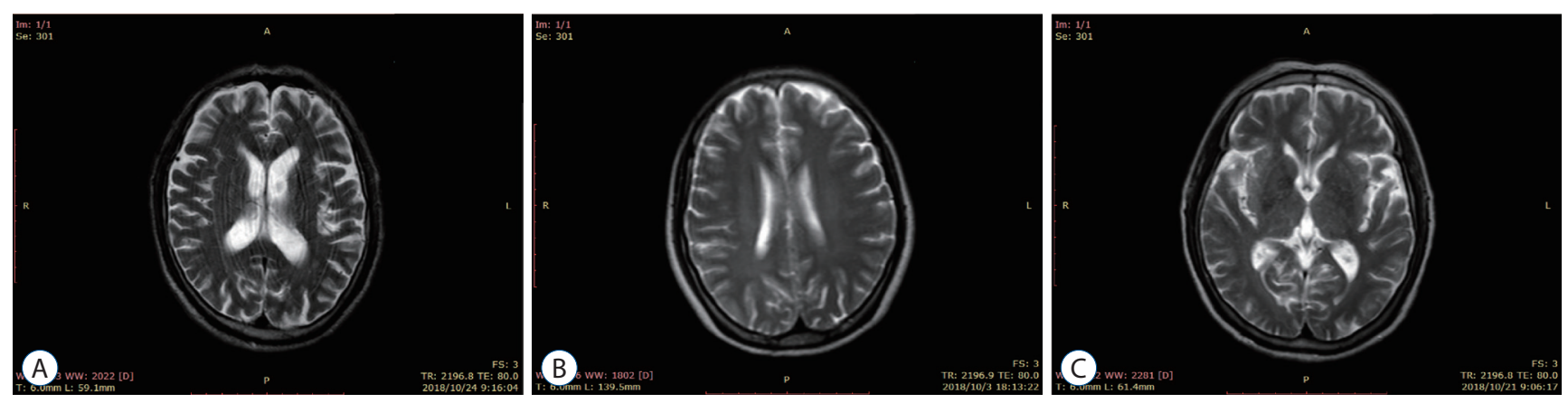

Fig. 2. Magnetic resonance imaging performance of cerebral small vessel disease. A : White matter lesions of brain. B : Cerebral microbleeds. C : The perivascular space is enlarged. 
the serum of the LI group increased $(p<0.05)$, and MLI combined with WML group increased $(p<0.001)$. Because the number of lesions in LI is not directly related to cerebral small vessels, it does not need to be considered (Table 1 and Fig. 3$)^{26)}$.

\section{Correlation analysis between serum miR-155 level and cerebrovascular disease}

The level of miR-155 in serum was positively correlated with the increase of imaging lesions $(p<0.05)$ (Table 1$)$.

\section{DISCUSSION}

\section{Changes and mechanisms of miR-155 levels in serum of patients with CSVD}

MiR-155 belongs to one of the miRs and is a typical multifunctional gene localized to human chromosome 21. Located

Table 1. MiR-155 levels in the three groups of serum and correlation analysis

\begin{tabular}{lcc}
\hline Group & N & Levels of serum miR-155 \\
\hline Control & 30 & $6.26 \pm 0.86$ \\
\hline LI & 8 & $11.52 \pm 1.62^{*}$ \\
MLI combined with WML & 22 & $15.82 \pm 1.25^{\dagger}$ \\
\hline F value & & 28.992 \\
P1 value & 0.000 \\
R value & 0.523 \\
P2 value & 0.042 \\
\hline
\end{tabular}

Values are presented as mean \pm standard deviation or number. ${ }^{*}$ Compared with the control group, $p<0.05 .{ }^{\dagger}$ Compared with the control group, $p<0.001$. miR-155 : microRNA-155, LI : lacunar infarction, MLI : multiple lacunar infarction, WML : white matter lesions

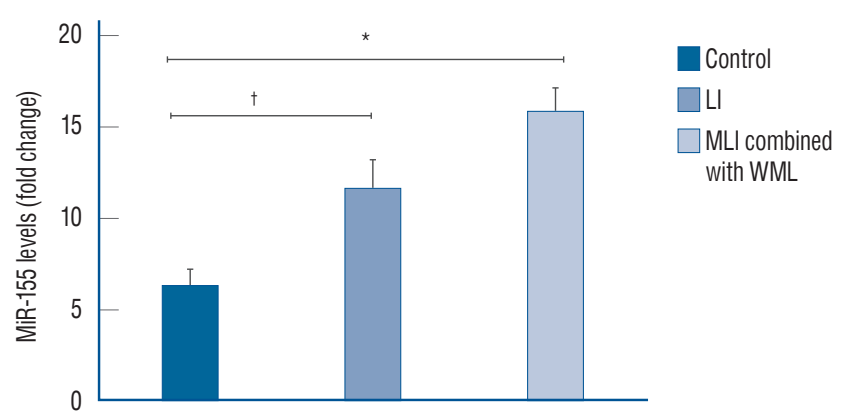

Fig. 3. MiR-155 levels in the three groups of serum (mean \pm standard deviation). ${ }^{*} p<0.01 .{ }^{\dagger} p<0.05$. miR-155 : microRNA-155, LI : lacunar infarction, MLI : multiple lacunar infarction, WML : white matter lesions. in the 3rd exon of the B-cell integration cluster (BIC) gene, its expression level is regulated by the transcriptional level of BIC gene and processing of other miRs.

This study ${ }^{15)}$ showed that the levels of miR-155 in the serum of LI group and MLI combined with WML were higher than those in the control group $(p<0.05)$, suggesting that the level of miR-155 in serum of CSVD increased ${ }^{29)}$. Further analysis of the correlation between the changes of serum miR-155 levels in patients with CSVD and the increase of imaging lesions found that the level of miR-155 in serum was positively correlated with the number of lesions $(p<0.05)$, showing that the more imaging lesions in patients with CSVD, the higher the level of miR-155 in the serum ${ }^{14)}$.

BBB dysfunction is considered to be a biological marker of cerebral stroke, and increased cerebral vascular permeability and $\mathrm{BBB}$ leakage will lead to ischemic brain damage ${ }^{1)}$. Zhang et al. ${ }^{34)}$ pointed out that miR-155 can be used as a negative regulator of $\mathrm{BBB}$ function. This study found that the inhibition of endogenous miR-155 attenuated some of the high permeability induced by cytokines ${ }^{2)}$. In addition, miR-155 not only targets cell-complex molecules, such as annexin-2 and annexin-1, but also targets adhesion molecules, such as DOCK-1 and Synthin-1 to regulate brain endothelial function. $\mathrm{Ago}^{1)}$ found that $\mathrm{Bcl}-2$ was an important anti-apoptotic protein in mice, which can reduce BBB destruction and cerebral infarction in mice after focal cerebral ischemia ${ }^{4}$.

\section{Significance of changes in serum miR-155 levels in patients with CVSD}

In recent years, a lot of research has shown that the maintenance and exercise of normal brain function mainly depend on the function and structure of sound and intact neurovascular units in the brain parenchyma ${ }^{1)}$. The integrity of neurovascular unit function depends on the neuroglial cells and neurons in the process of neurotransmitter conversion and transmission $^{19)}$, and then coordinates with each other to perform their functions; the integrity of neurovascular unit structure depends on the integrity of its multiple constituent cells-nerve and non-neural cell integrity ${ }^{30)}$. Neurons and glial cells are involved in the formation of nerve cells, and glial cells contain microglia, astrocytes, and oligodendrocytes; nonneuronal cells include vascular smooth muscle cells and vascular endothelial cells, basement membrane and extracellular matrix $^{10)}$. Although the brain parenchymal damage caused by 
CSVD is small, it also destroys the function and structural integrity of the neurovascular unit.

Nitric oxide (NO) secreted by vascular endothelium is for vascular endothelial homeostasis. Decreased NO released by nitricoxide synthase leads to endothelial dysfunction. Hypoxia and ischemia reduce endothelial nitric oxide synthetase (eNOS) expression in endothelial cells through post-transcriptional mechanism, resulting in NOS3 transcriptional instability. In this study, we found that miRNA contributes to this mechanism $^{8)}$. By studying changes in the expression profile of miRs in ischemic stroke, it was found that the expression level of miR-155 in rat brain tissue was increased ${ }^{36)}$, indicating that miR-155 was closely related to ischemic stroke. In recent years, studies have shown that in the acute phase of stroke in mice, the inhibition of miR-155 protects cerebral vascular endothelial cells ${ }^{12)}$, attenuates immune inflammatory responses in ischemic mouse brain tissue ${ }^{16)}$, and is good for neurological rehabilitation and infarct size reduction in ischemic stroke mice $^{27)}$. Pena-Philippides research team used novel Locked Nucleic Acid technology ${ }^{36)}$ to inhibit miR-155 in ischemic stroke mice and found that the expressions of cytokines CCL12, CX-CL3 and inflammation-related molecules were significantly reduced in experimental mice. It promotes cerebral angiogenesis, reduces brain tissue damage, and improves neurological function recovery. In the mouse model of stroke, there are also studies that use acetylcholine ester to inhibit miR-155 and achieve good recovery ${ }^{32}$. The new treatments in these experiments will provide new ideas for the treatment of clinical patients, and further clinical trials are needed to confirm this result ${ }^{6)}$.

The level of miR-155 in serum plays a role in the occurrence, development and prognosis of CSVD ${ }^{9)}$. The increase of serum miR-155 level in patients with CSVD may be one of its selfprotection mechanisms, and the strength of this self-protection mechanism may be positively correlated with the number of lesions. Further in-depth research on the level of miR-155 in the serum of CSVD will bring new ways and methods for the prevention and treatment of $\mathrm{CSVD}^{20)}$. It is hoped that more researchers will contribute to the clearer relationship between miR-155 and inflammatory markers from different aspects, and further study of relationship between miR-155 and cerebral small vessels.

\section{CONCLUSION}

This study shows that the change of serum miR-155 level in CSVD patients may be one of the self-protection mechanisms, and the intensity of this self-protection mechanism is positively correlated with the number of CSVD lesions. It provides another idea for the diagnosis and treatment of CSVD.

\section{CONFLICTS OF INTEREST}

No potential conflict of interest relevant to this article was reported.

\section{INFORMED CONSENT}

Informed consent was obtained from all individual participants included in this study.

\section{AUTHOR CONTRIBUTIONS}

\author{
Conceptualization : YG \\ Data curation : YG, DL, JL, NY, DW \\ Formal analysis : YG, DL, JL, NY, DW \\ Funding acquisition : YG \\ Methodology : YG \\ Visualization : YG \\ Writing - original draft : $Y G$ \\ Writing - review \& editing : YG
}

\section{ORCID}

$\begin{array}{ll}\text { Ying Guo } & \text { https://orcid.org/0000-0001-7448-4552 } \\ \text { Dongxue Li } & \text { https://orcid.org/0000-0001-9073-1242 } \\ \text { Jiapei Li } & \text { https://orcid.org/0000-0001-9318-9647 } \\ \text { Nan Yang } & \text { https://orcid.org/0000-0003-1019-3435 } \\ \text { Deyun Wang } & \text { https://orcid.org/0000-0002-4171-5075 }\end{array}$

\section{- Acknowledgements}

The authors thank Guangzhou Yujia Biotechnology Co., 
Ltd for excellent technical assistance.

\section{References}

1. Ago $T$ : The neurovascular unit in health and ischemic stroke. Nihon Rinsho 74 : 583-588, 2016

2. Arai K, Lok J, Guo S, Hayakawa K, Xing C, Lo EH : Cellular mechanisms of neurovascular damage and repair after stroke. J Child Neurol 26 : 1193-1198, 2011

3. Cerebrovascular disease group of Neurology branch of Chinese Medical Association; Neurology Branch of Chinese Medical Association : Consensus on diagnosis and treatment of cerebrovascular disease in China. Chin J Neurol 48 : 838-844, 2015

4. Chiu AP, Wan A, Lal N, Zhang D, Wang F, Vlodavsky I, et al. : Cardiomyocyte VEGF regulates endothelial cell GPIHBP1 to relocate lipoprotein lipase to the coronary lumen during diabetes mellitus. Arterioscler Thromb Vasc Biol 36 : 145-155, 2016

5. Cuadrado-Godia E, Dwivedi P, Sharma S, Ois Santiago A, Roquer Gonzalez J, Balcells $M$, et al. : Cerebral small vessel disease: a review focusing on pathophysiology, biomarkers, and machine learning strategies. J Stroke 20 : 302-320, 2018

6. Duan $\mathrm{CL}$, Liu CW, Shen SW, Yu Z, Mo JL, Chen XH, et al. : Striatal astrocytes transdifferentiate into functional mature neurons following ischemic brain injury. Glia 63 : 1660-1670, 2015

7. Garzon R, Croce CM : MicroRNAs in normal and malignant hematopoiesis. Curr Opin Hematol 15 : 352-358, 2008

8. Janaszak-Jasiecka A, Siekierzycka A, Bartoszewska S, Serocki M, Dobrucki LW, Collawn JF, et al. : eNOS expression and NO release during hypoxia is inhibited by miR-200b in human endothelial cells. Angiogenesis $21: 711-724,2018$

9. Kawasaki Y, Fujiki M, Uchida S, Morishige M, Momii Y, Ishii K : A single oral dose of geranylgeranylacetone upregulates vascular endothelial growth factor and protects against Kainic acid-induced neuronal cell death: involvement of the phosphatidylinositol-3 kinase/Akt pathway. Pathobiology 84 : 184-191, 2017

10. Latzer $P$, Schlegel $U$, Theiss $C$ : Morphological changes of cortical and hippocampal neurons after treatment with VEGF and bevacizumab. CNS Neurosci Ther 22 : 440-450, 2016

11. Leng RX, Pan HF, Qin WZ, Chen GM, Ye DQ : Role of microRNA-155 in autoimmunity. Cytokine Growth Factor Rev 22 : 141-147, 2011

12. Lopez-Ramirez MA, Wu D, Pryce G, Simpson JE, Reijerkerk A, KingRobson J, et al. : MicroRNA-155 negatively affects blood-brain barrier function during neuroinflammation. FASEB J 28 : 2551-2565, 2014

13. Markus HS, Hunt B, Palmer $K$, Enzinger $C$, Schmidt H, Schmidt R : Markers of endothelial and hemostatic activation and progression of cerebral white matter hyperintensities: Iongitudinal results of the Austrian Stroke Prevention Study. Stroke 36 : 1410-1414, 2005

14. McConnell HL, Kersch CN, Woltjer RL, Neuwelt EA : The translational significance of the neurovascular unit. J Biol Chem 292 : 762-770,
2017

15. Mizushima N, Komatsu M : Autophagy: renovation of cells and tissues. Cell 147 : 728-741, 2011

16. Pan ZG, Mao Y, Sun FY : VEGF enhances reconstruction of neurovascular units in the brain after injury. Sheng Li Xue Bao 69 : 96-108, 2017

17. Pantoni $\mathrm{L}$ : Cerebral small vessel disease: from pathogenesis and clinical characteristics to therapeutic challenges. Lancet Neurol 9 : 689-701, 2010

18. Poggesi A, Pasi M, Pescini F, Pantoni L, Inzitari D : Circulating biologic markers of endothelial dysfunction in cerebral small vessel disease: a review. J Cereb Blood Flow Metab 36 : 72-94, 2016

19. Shen SW, Duan CL, Chen XH, Wang YQ, Sun X, Zhang QW, et al. : Neurogenic effect of VEGF is related to increase of astrocytes transdifferentiation into new mature neurons in rat brains after stroke. Neuropharmacology 108 : 451-461, 2016

20. Silva-Hucha S, Hernández RG, Benítez-Temiño B, Pastor ÁM, de la Cruz RR, Morcuende $S$ : Extraocular motoneurons of the adult rat show higher levels of vascular endothelial growth factor and its receptor Flk-1 than other cranial motoneurons. PLoS One 12 : e178616, 2017

21. Sochor M, Basova P, Pesta M, Dusilkova N, Bartos J, Burda P, et al. : Oncogenic microRNAs: miR-155, miR-19a, miR-181b, and miR-24 enable monitoring of early, breast cancer in serum. BMC Cancer $14: 448$, 2014

22. Staszel T, Zapala B, Polus A, Sadakierska-Chudy A, Kieć -Wilk B, Stępień $E$, et al. : Role of microRNAs in endothelial cell pathophysiology. Pol Arch Med Wewn $121: 361-366,2011$

23. Tang MM, Lin WJ, Pan YQ, Guan XT, Li YC : Hippocampal neurogenesis dysfunction linked to depressive-like behaviors in a neuroinflammation induced model of depression. Physiol Behav 161 : 166-173, 2016

24. Teng G, Papavasiliou FN : Shhh! Silencing by microRNA-155. Philos Trans R Soc Lond B Biol Sci 364 : 631-637, 2009

25. Wardlaw JM, Sandercock PA, Dennis MS, Starr J : Is breakdown of the blood-brain barrier responsible for lacunar stroke, leukoaraiosis, and dementia? Stroke 34 : 806-812, 2003

26. Wardlaw JM, Smith C, Dichgans M : Mechanisms of sporadic cerebral small vessel disease: insights from neuroimaging. Lancet Neurol 12 : 483-497, 2013

27. Wu KW, Yang P, Li SS, Liu CW, Sun FY : VEGF attenuated increase of outward delayed-rectifier potassium currents in hippocampal neurons induced by focal ischemia via PI3-K pathway. Neuroscience 298 : 94 101, 2015

28. Wu Q, Jin H, Yang Z, Luo G, Lu Y, Li K, et al. : MiR-150 promotes gastric cancer proliferation by negatively regulating the pro-apoptotic gene EGR2. Biochem Biophys Res Commun 392 : 340-345, 2010

29. Xie XG, Zhang M, Dai YK, Ding MS, Meng SD : Combination of vascular endothelial growth factor-loaded microspheres and hyperbaric oxygen on random skin flap survival in rats. Exp Ther Med 10 : 954-958, 2015

30. Yang J, Yang C, Liu C, Zhang T, Yang Z : Paradoxical effects of VEGF on synaptic activity partially involved in notch1 signaling in the mouse hippocampus. Hippocampus 26 : 589-600, 2016 
Relationship between miR-155 and CSVD | Guo Y, et al.

31. Yassi $\mathrm{N}$, Desmond PM, Masters $\mathrm{CL}$ : Magnetic resonance imaging of vascular contributions to cognitive impairment and dementia. J Mol Neurosci 60 : 349-353, 2016

32. Yin KJ, Deng Z, Hamblin M, Xiang Y, Huang $H$, Zhang J, et al. : Peroxisome proliferator-activated receptor delta regulation of miR-15a in ischemia-induced cerebral vascular endothelial injury. J Neurosci 30 : 6398-6408, 2010

33. Zhang CM, Zhao J, Deng HY : MiR-155 promotes proliferation of human breast cancer MCF-7 cells through targeting tumor protein 53-induced nuclear protein 1. J Biomed Sci 20 : 79, 2013

34. Zhang J, Zou H, Zhang Q, Wang L, Lei J, Wang Y, et al. : Effects of Xiaoshuan enteric-coated capsule on neurovascular functions assessed by quantitative multiparametric MRI in a rat model of permanent cerebral ischemia. BMC Complement Altern Med 16 : 198, 2016

35. Zhang L, Wang W, Li X, He S, Yao J, Wang X, et al. : MicroRNA-155 promotes tumor growth of human hepatocellular carcinoma by targeting ARID2. Int J Oncol 48 : 2425-2434, 2016

36. Zhou ZC, Gu SZ, Wu J, Liang QW : VEGF, eNOS, and ABCB1 genetic polymorphisms may increase the risk of osteonecrosis of the femoral head. Genet Mol Res 14 : 13688-13698, 2015

37. Zhu X, Wang Y, Sun Y, Zheng J, Zhu D : MiR-155 up-regulation by LMP1 DNA contributes to increased nasopharyngeal carcinoma cell proliferation and migration. Eur Arch Otorhinolaryngol 271 : 1939-1945, 2014 